\title{
Prevalencia de disfunción sexual femenina en un hospital de tercer nivel de la Ciudad de México
}

\section{Prevalence of female sexual dysfunction at a tertiary care hospital in Mexico City}

Roberto Patricio López-Maguey, Mauricio Cantellano-Orozco, Josette Rosana Staufert-Gutiérrez, José GómezSánchez, Javier Antonio Herrera-Muñoz, Diego Antonio Preciado-Estrella y colaboradores

\begin{abstract}
Resumen
OBJETIVO: Estimar la prevalencia de disfunción sexual femenina en un hospital de tercer nivel de atención de la Ciudad de México.

MATERIALES Y MÉTODOS: Estudio prospectivo y descriptivo, al que se incluyeron mujeres de 20 a 60 años de edad, quienes asistieron a consulta o formaron parte del equipo médico y de trabajo del Hospital General Dr. Manuel Gea González, entre noviembre de 2016 y junio de 2017, para responder el Cuestionario IFSF (por sus siglas en inglés). La disfunción sexual se calculó para cada dominio con un puntaje menor a 3, clasificándose como función sexual normal al puntaje mayor de 28 y disfunción sexual al puntaje menor a éste. Para el análisis estadístico se utilizó el programa SPSS, versión 1.0.0, además del ANOVA y t de Student para la comparación entre los grupos y dominios. RESULTADOS: Se aplicaron 171 cuestionarios; el promedio de edad de las participantes fue de 34.6 años; 1.1 parejas sexuales en los últimos cuatro meses e IMC de $27.7 \mathrm{~kg}$. Solo $45(35 \%)$ mujeres disfunción sexual. La prevalencia aumentó de acuerdo con la edad: en menores de 30 años fue de $17 \%$ y en mayores de 40 de $66 \%$. Los principales trastornos asociados con la disfunción sexual fueron: deseo sexual disminuido (28\%), incapacidad para la excitación (17\%), para alcanzar el orgasmo $(17 \%)$, para conseguir la lubricación suficiente (11\%) y dispareunia (11\%).

CONCLUSIONES: La prevalencia de disfunción sexual femenina fue similar a la reportada en la bibliografía mundial (35 vs 38\%). Aunque se reportó un alto índice de obesidad, no se correlacionó con la disfunción sexual femenina.

PALABRAS CLAVE: Disfunción sexual femenina; cuestionario IFSF; dispareunia.

Abstract

OBJECTIVE: To estimate the prevalence of female sexual dysfunction at a tertiary care hospital in Mexico City.

MATERIALS AND METHODS: A prospective and descriptive study in which the Index of Female Sexual Function (IFSF) questionnaire was applied to women between 20 and 60 years of age that were in medical consultation, were part of the medical team, or belonged to the work force at the Hospital Dr. Manuel Gea González, within the time frame of November 2016 and June 2017. Sexual dysfunction was calculated for each domain with a score under 3 . Normal sexual function was classified by a score above 28 and sexual dysfunction by a score below 28. The SPSS version 1.0.0 was used for the statistical analysis and group and domain comparisons were performed with the ANOVA test and the Student's t test.

RESULTS: A total of 171 questionnaires were applied. Mean participant age was 34.6 years, number of sexual partners within the last 4 months was 1.1 , and BMI was 27.7 $\mathrm{kg}$. Only $45(35 \%)$ women presented with female sexual dysfunction and its prevalence increased in relation to age: it was $17 \%$ in women under 30 years of age and $66 \%$ in
\end{abstract}

División de Urología, Hospital General Dr. Manuel Gea González, Ciudad de México.

Recibido: octubre 2017

Aceptado: abril 2018

Correspondencia

Roberto Patricio López Maguey rpatloma@gmail.com

Este artículo debe citarse como López-Maguey RP, Cantellano-Orozco M, Staufert-Gutiérrez JR, Gómez-Sánchez J, Herrera-Muñoz JA, Preciado-Estrella DA y col. Prevalencia de disfunción sexual femenina en un hospital de tercer nivel de la Ciudad de México. Rev Mex Urol. 2018 mayo-junio;78(3):169-175. DOI:https://doi.org/10.24245/revmexurol.v78i3.1747 
women above 40 years of age. The main disorders associated with sexual dysfunction were: reduced sexual desire (28\%), inability to achieve orgasm $(17 \%)$ or arousal $(17 \%)$, deficient lubrication $(11 \%)$, and dyspareunia $(11 \%)$.

CONCLUSIONS: The prevalence of female sexual dysfunction was similar to that reported in the international literature (35 vs 38\%). Even though a high rate of obesity was reported, it did not correlate with female sexual dysfunction.

KEYWORDS: Female sexual dysfunction; Index of Female Sexual Function (IFSF); Dyspareunia.

\section{ANTECEDENTES}

La disfunción sexual es una alteración que suele afectar a mujeres de edad media (mayores de 40 años), con principal repercusión en la calidad de vida, sobre todo en la autoestima, integridad de la persona y relaciones interpersonales. México no cuenta con reportes epidemiológicos acerca de este padecimiento; sin embargo, algunos estudios muestran una prevalencia de 38 a $63 \%$, lo que provoca un efecto social importante. ${ }^{1,2}$

La definición de disfunción sexual femenina se adoptó durante el Cuarto Consenso Internacional de Medicina Sexual (ICSM), Ilevado a cabo en junio de 2015. El origen de la disfunción sexual femenina puede ser desconocido o multifactorial. Comúnmente se ve afectado el deseo, la excitación, el orgasmo, la lubricación o satisfacción sexual. La mayor parte de las clasificaciones que establecen el diagnóstico de disfunción sexual femenina son descriptivas. ${ }^{3,4}$

Aunque se han propuesto diferentes escalas para establecer el diagnóstico de disfunción sexual femenina, hoy día las más utilizadas son la Clasificación Internacional de Enfermedades (10 edición; ICD-10), avalada por la Organización Mundial de la Salud, y el Manual de Desórdenes Mentales, Diagnóstico y Estadística ( $4^{\mathrm{a}}$ y 5 a ediciones, DSM-1V-TR y DSM-5, respectivamente), aprobado por la asociación Americana de Psiquiatría. Estos cuestionarios dividen las alteraciones en causas orgánicas (vaginismo y dispareunia) y no orgánicas (falta de deseo o aversión sexual; placer disminuido, falla de respuesta genital, disfunción orgásmica, vaginismo no orgánico, dispareunia no orgánica e impulso sexual desmedido). ${ }^{5,6}$

Para establecer el diagnóstico de disfunción sexual femenina, la Clasificación DSM-5 requiere la manifestación de síntomas por lo menos durante 6 meses, en aproximadamente $75 \%$ de las relaciones, que causen un estrés significativo y no sea consecuencia de algún desorden mental o cierta relación interpersonal disfuncional, incluso que se atribuya a un medicamento. Además, debe diferenciarse si la alteración ha coexistido:

- Durante toda la vida (la anormalidad inició desde que la persona es sexualmente activa) o fue adquirida (comenzó posterior a un periodo relativamente normal de sexualidad).

- Generalizada (no limitada a ciertos tipos de estimulación, situación o pareja) o situacional (ocurre solamente con ciertas estimulaciones, situaciones o parejas).

- La manifestación de síntomas en los últimos 6 meses ha sido mínima, moderada o severa.

Con base en lo anterior, el objetivo de este estudio fue estimar la prevalencia de disfunción sexual femenina en un hospital de tercer nivel de atención de la Ciudad de México. 


\section{MATERIALES Y MÉTODOS}

Estudio prospectivo y descriptivo, al que se incluyeron mujeres de 20 a 60 años de edad, quienes asistieron a consulta o formaron parte del equipo médico y de trabajo del Hospital General Dr. Manuel Gea González, entre noviembre de 2016 y junio de 2017, para responder el Cuestionario IFSF de disfunción sexual femenina. Todas las participantes dieron su consentimiento informado antes de la entrevista. Se eliminaron del estudio las mujeres que no respondieron completamente el cuestionario, quienes no tuvieron actividad sexual en el último mes o se encontraran en la posmenopausia. Se dividieron por grupos de edad en: 20-29, 30-39, 40-49 y más de 50 años, para aplicar un cuestionario estándar demográfico y analizar la edad, estado civil, número de parejas sexuales en los últimos 4 meses, escolaridad, tabaquismo, alcoholismo e índice de masa corporal (IMC).

La disfunción sexual femenina se evaluó mediante el cuestionario IFSF, ${ }^{4}$ que valora la función o alteraciones sexuales en las últimas cuatro semanas. De acuerdo con el IFSF, se incluyen los dominios de deseo, excitación, lubricación, orgasmo, satisfacción y dolor (dispareunia) durante la relación sexual. El deseo sexual se evaluó de acuerdo con la frecuencia y el nivel de deseo en dos preguntas; la excitación se refirió según la frecuencia, nivel de confianza y satisfacción en 4 preguntas. ${ }^{5}$ La lubricación se determinó con el grado de frecuencia y dificultad, además de la frecuencia y dificultad para mantenerlas, en 4 preguntas. El orgasmo se evaluó con 3 preguntas que incluyen frecuencia, dificultad y satisfacción. La satisfacción de la relación se refiere a la cercanía con la pareja, la relación sexual y vida sexual en general. La dispareunia se evaluó de acuerdo con su manifestación durante la penetración vaginal, frecuencia y grado. En total se plantean 19 preguntas divididas entre los rubros, con un valor de 1 a 5 puntos..$^{8-10}$
La disfunción sexual se calculó para cada dominio con un puntaje menor a 3, clasificándose como función sexual normal al puntaje mayor de 28 y disfunción sexual al puntaje menor a éste.

Para el análisis estadístico se utilizó el programa SPSS, versión 1.0.0, además del ANOVA y t de Student para la comparación entre los grupos y dominios.

\section{RESULTADOS}

Durante el estudio se aplicaron 200 encuestas; sin embargo, solo se aprobaron 171 (85.5\%). Las características demográficas de la población se muestran en el Cuadro 1. La edad media registrada fue de $34.6 \pm 11$ años, número de parejas sexuales de 1.1 e IMC de $27.48 \pm 6.63 \mathrm{~kg} / \mathrm{m}^{2}$, con mayor predominio de pacientes con sobrepeso $(\mathrm{n}=89,52 \%)$. En cuanto a toxicomanías, $61(35 \%)$ mujeres refirieron tabaquismo y 71 (45\%) alcoholismo. Las comorbilidades identificadas con mayor frecuencia fueron: ansiedad, depresión e hipotiroidismo. En relación con la escolaridad, 100 (58\%) mujeres contaban con licenciatura y $59 \%$ estaban solteras.

La prevalencia de disfunción sexual aumentó conforme a la edad, principalmente en el grupo de 40-49 años, con un total de 14 (43\%) mujeres (Cuadro 2).

El Cuadro 3 expone la media del puntaje por cada dominio de la encuesta, dividido por grupos de edad. La única variable con diferencia estadísticamente significativa fue el deseo sexual $(p=0.001)$. En cuanto a los demás dominios, la disfunción sexual se encontró en 48 (28\%) mujeres; deficiente excitación en 29 (17\%) y lubricación disminuida en 18 (11\%) casos; disfunción del orgasmo en 29 (17\%), alteración de la satisfacción sexual en $22(13 \%)$ y dispareunia en $18(11 \%)$ mujeres. En total se registraron 45 (35\%) mujeres con disfunción sexual. 
Cuadro 1. Características de las participantes

\begin{tabular}{|c|c|}
\hline Variable & Valor $(n=171)$ \\
\hline Edad promedio & $34.65 \pm 11$ \\
\hline Parejas sexuales promedio & $1.1 \pm 0.49$ \\
\hline \multicolumn{2}{|l|}{ Estado civil } \\
\hline Solteras & $102(59 \%)$ \\
\hline Casadas & $60(35 \%)$ \\
\hline Unión libre & $7(4 \%)$ \\
\hline Viudas & $2(2 \%)$ \\
\hline \multicolumn{2}{|l|}{ Escolaridad } \\
\hline Licenciatura & $100(58 \%)$ \\
\hline Posgrado (maestría) & $25(14.6 \%)$ \\
\hline Preparatoria & $37(21.3 \%)$ \\
\hline Secundaria & $6(3.5 \%)$ \\
\hline Primaria & $3(1.7 \%)$ \\
\hline \multicolumn{2}{|l|}{ IMC } \\
\hline Sobrepeso (IMC 25-29) & $89(52.04 \%)$ \\
\hline Obesidad (IMC>30) & $54(31.5 \%)$ \\
\hline Normal (IMC18-24) & $28(16.3 \%)$ \\
\hline \multicolumn{2}{|l|}{ Comorbilidades } \\
\hline Ansiedad & 9 \\
\hline Asma & 2 \\
\hline Cardiaca & 1 \\
\hline Colitis & 3 \\
\hline Depresión & 6 \\
\hline Hipertensión & 1 \\
\hline Dermatitis & 2 \\
\hline Hipotiroidismo & 8 \\
\hline
\end{tabular}

Cuadro 2. Prevalencia de disfunción sexual femenina por grupos de edad

\begin{tabular}{|l|c|c|}
\hline Grupo de edad & Disfunción & Sin disfunción \\
\hline $20-29$ años & $12(17 \%)$ & $57(83 \%)$ \\
\hline $30-39$ años & $14(28 \%)$ & $35(72 \%)$ \\
\hline $40-49$ años & $14(43 \%)$ & $18(56 \%)$ \\
\hline 50 años & $5(5 \%)$ & $16(77 \%)$
\end{tabular}

En el grupo de 20-29 años de edad se encontraron 9 mujeres con disfunción sexual, de las que 2 tuvieron trastorno de ansiedad, 1 dermatitis atópica y 6 obesidad, con escolaridad de secundaria $(n=1)$, nivel medio superior $(n=2)$ y licenciatura $(n=6)$. En el grupo de 30-39 años se registró un 1 caso de asma, 1 de hipercolesterolemia, 1 de hipotiroidismo y 1 de obesidad. En el grupo de 40-49 años, 1 paciente manifestó hipotiroidismo, 1 depresión, 1 ansiedad y 5 padecían obesidad. En el grupo de más de 50 años se encontró 1 mujer con diabetes, 1 con depresión y 2 con obesidad.

\section{DISCUSIÓN}

La disfunción sexual femenina en urología es un tema poco estudiado. El ensayo de PérezMartínez y su grupo, realizado en Chihuahua en 2006, en el que aplicaron el IFSF a 44 pacientes de 17 a 49 años de edad, reportó una prevalencia de $34 \%$ de disfunción sexual femenina, cuyos

Cuadro 3. Resultados de dominios por grupos de edad

\begin{tabular}{|c|c|c|c|c|c|c|c|}
\hline Parámetros & $\begin{array}{c}20-29 \\
(n=69)\end{array}$ & $\begin{array}{c}30-39 \\
(n=49)\end{array}$ & $\begin{array}{c}40-49 \\
(n=32)\end{array}$ & $\begin{array}{c}>50 \\
(n=21)\end{array}$ & p & $\begin{array}{c}\text { Promedio total del } \\
\text { puntaje ( } \pm \text { DS) }\end{array}$ & Total \\
\hline Deseo & $3.9 \pm 1.4$ & $6.5 \pm 1.2$ & $4.36 \pm 1.4$ & $3.4 \pm 1.5$ & 0.001 & $2.1 \pm 1.01$ & $47(28 \%)$ \\
\hline Excitación & $3.33 \pm 1.8$ & $3.71 \pm 1.7$ & $4.14 \pm 1.6$ & $4.20 \pm 1.9$ & 0.11 & $3.57 \pm 1.8$ & $29(17 \%)$ \\
\hline Lubricación & $4.83 \pm 1.7$ & $4.22 \pm 1.9$ & $3.36 \pm 1.8$ & $4.13 \pm 1.7$ & 0.8 & $3.73 \pm 1.9$ & $18(11 \%)$ \\
\hline Orgasmo & $3.42 \pm 1.4$ & $5.44 \pm 1.6$ & $5.36 \pm 1.9$ & $3.70 \pm 1.7$ & 0.257 & $2.72 \pm 1.95$ & $29(17 \%)$ \\
\hline Satisfacción & $4.08 \pm 1.9$ & $3.86 \pm 1.8$ & $2.64 \pm 1.7$ & $3.3 \pm 1.9$ & 0.445 & $3.08 \pm 2.5$ & $22(13 \%)$ \\
\hline $\begin{array}{l}\text { Dispareunia } \\
\text { (dolor) }\end{array}$ & $5.25 \pm 1.8$ & $2.5 \pm 1.9$ & $2.07 \pm 1.4$ & $3.5 \pm 1.8$ & 0.356 & $3.2 \pm 1.25$ & $18(11 \%)$ \\
\hline Global & $24.81 \pm 5.5$ & $26.16 \pm 4.5$ & $21.93 \pm 5.4$ & $22.23 \pm 5.7$ & 0.01 & $18.11 \pm 4.6$ & 45 (35\%) \\
\hline
\end{tabular}


trastornos más frecuentes fueron: deseo sexual disminuido (20.4\%), deficiente excitación sexual (27.7\%), anomalías en la lubricación (34\%) y anorgasmia (18.8\%). Entre los factores más importantes para padecer disfunción sexual se encontraron la edad (34.5 años) y el estado civil. ${ }^{2}$

En este estudio no comparamos los factores de riesgo asociados con la deficiencia sexual femenina; sin embargo, se observó que el deseo sexual fue la disfunción más común, quizá debido a múltiples situaciones, como lo expone el estudio de Wen-Jia y sus coautores, ${ }^{11}$ quienes evaluaron a 4697 pacientes de 20 a 60 años de edad y encontraron una prevalencia de disfunción sexual de $63.3 \%$. Entre los factores de riesgo reportaron: insatisfacción con la pareja, pobre afecto en el matrimonio, imposibilidad por parte del esposo, vivir en una zona rural, bajo nivel de educación y estado posmenopáusico. Los trastornos más frecuentes fueron: dificultad para lograr la excitación (80\%), alteraciones en la lubricación (32.4\%), disfunción orgásmica (29.9\%) y dispareunia (31.6\%). ${ }^{9}$ También se observó que mientras más alto es el nivel de educación existe mayor preocupación por la satisfacción sexual y lograr orgasmos. Con base en esto, es más difícil identificar ciertos factores de riesgo en mujeres con bajo nivel de educación.

McCool y sus colaboradores ${ }^{12}$ realizaron un metanálisis entre 2000 y 2014 de prevalencia de disfunción sexual y encontraron 135 artículos, con reportes de Europa, Estados Unidos, Egipto, Marruecos, Brasil, Malasia, Turquía, China, Japón y Tailandia. La prevalencia estimada en esos países fue de $40.9 \%$ y los trastornos más comunes fueron la dificultad de lubricación (20.6\%) y deseo sexual hipoactivo (28.2\%). También observaron que a mayor grado de estudios, las mujeres se encontraban más conscientes de identificar y reportar los trastornos sexuales, además de relacionarse con mayor decepción de las relaciones sexuales. ${ }^{10}$
Otro de los factores con mayor riesgo de disfunción sexual es la obesidad. Rabiepoor y su grupo $^{13}$ efectuaron un estudio en mujeres iraníes y reportaron esta alteración en pacientes con sobrepeso y obesidad. El estudio incluyó 200 pacientes de 29 años de edad (17 a 45 años), con nivel de estudios de licenciatura y al menos 5 años de matrimonio, y encontraron como trastornos más comunes la disfunción orgásmica, excitación y dispareunia. El 69.7\% refirió baja satisfacción o insatisfacción y solo $30.3 \%$ se encontraba satisfecha. ${ }^{11}$ Aunque en nuestro estudio no encontramos una relación importante con este trastorno, la mayoría de las mujeres tenía sobrepeso $(51.3 \%$ padecía obesidad considerable); sin embargo, si la muestra fuera más grande tendríamos una correlación con la obesidad.

Diversos estudios señalan elevada prevalencia de disfunción sexual en mujeres de 40 a 65 años de edad; por lo tanto, es importante aplicar cuestionarios de calidad de vida en pacientes menopáusicas, pues representa un problema que va en aumento en México. Worsley y su equipo de trabajo, ${ }^{14}$ quienes evaluaron a 2020 mujeres australianas mediante el IFSF, establecieron el diagnóstico de disfunción sexual en las pacientes que obtuvieron puntuaciones menores de 5. Así mismo, agregaron otras escalas, como el Cuestionario de Calidad de Vida en la Menopausia, aplicado a pacientes de entre 51 y 65 años, y encontraron una prevalencia de $69.3 \%$, incluso reportaron que $69.7 \%$ mostró deseo sexual disminuido, sobre todo las mujeres que no tenían pareja sexual, mientras que quienes tenían pareja, esta variable fue de $75 \%$, con mayor incidencia en mujeres de 60 a 65 años. Los factores de riesgo más comunes para disfunción sexual fueron, después de la edad, la resequedad vaginal, síntomas depresivos y consumo de alcohol. ${ }^{7}$ En nuestro estudio, al comparar la estabilidad de la pareja y el consumo de bebidas alcohólicas, no encontramos una relación significativa en este rubro. Casi todos los estudios sugieren 
que la mayoría de las mujeres con disfunción sexual son casadas o viven en matrimonio, lo que contrasta con nuestra investigación, donde a pesar de tener un mayor registro de soltería, no se encontró afectada la disfunción sexual.

Jaafarpour y sus coauotres ${ }^{1}$ realizaron un estudio para estimar la prevalencia e identificar los factores de riesgo de disfunción sexual en mujeres iraníes. Seleccionaron 400 pacientes de 18 a 50 años de edad y encontraron una prevalencia de disfunción sexual de $22 \%$ en mujeres menores de 20, mientras que en pacientes de 40 a 50 años reportaron $75.7 \%$. Los trastornos más comunes fueron: lubricación deficiente (41.2\%), dificultad para la excitación $(37.5 \%)$, alteraciones para lograr el orgasmo (42\%) y dispareunia $(42.5 \%)$. Lo anterior se reportó, principalmente, en mujeres mayores de 40 años, quienes experimentan menos de tres relaciones sexuales a la semana. ${ }^{1}$ Al igual que en todo el mundo, nuestro estudio también sugiere que la prevalencia de disfunción aumenta conforme mayor es la edad; sin embargo, no todo se debe a la menopausia, sino a problemas multifactoriales. Por lo tanto, esta alteración debe estudiarse con mayor amplitud, para tener un efecto positivo en la salud de las pacientes. ${ }^{15-18}$

\section{CONCLUSIONES}

La prevalencia de disfunción sexual femenina fue similar a la reportada en la bibliografía mundial. El trastorno más común fue la disminución del deseo sexual y, aunque en nuestra población se reportó un alto índice de obesidad, no se correlacionó directamente con la disfunción sexual. Se requieren estudios adicionales que evalúen con mayor profundidad este tema.

\section{Colaboradores}

Gustavo Adolfo Veliz-Cabrera, Ulises Cristóbal Sánchez-Aquino, Guillermo Viana-Álvarez,
Pascual Cortes-Raygoza, Iván Calvo-Vázquez, Erick Hernández-Méndez, Mario OrtegaGonzález, Carlos Martínez-Arroyo, Gerardo Fernández-Noyola, Gustavo Morales-Montor, Carlos Pacheco-Gahbler

\section{REFERENCIAS}

1. Jaafarpour M, Khajavikhan J, Suhrabi Z. Female sexual dysfunction: prevalence and risk factors. J Clin Diag Res 2013;7(12):2877-2880.

2. Pérez-Martínez C, Vargas-Díaz HB, Castolo M. Prevalencia de disfunción sexual femenina en una población que asiste a una unidad de urología. Rev Mex Urol 2008;68(2):98-102.

3. McCabe M, Sharlip I, Elham A, et al. Definitions of sexual dysfunctions in women and men: a consensus statement from the Fourth International Consultation on Sexual Medicine 2015. J Sex Med 2016;13:135-143.

4. Levin R, Both S, Georgiadis, et al. The physiology of female sexual function and the pathophysiology of female sexual dysfunction. (Committee 13A). J Sex Med 2016;13:733-759.

5. Parish S, Goldstein AT, Goldstein SW, et al. Toward a more evidence-based nosology and nomenclature for female sexual dysfunctions-Part II. J Sex Med 2016;13(12):18881906.

6. Famer $\mathrm{M}$, Yoon $\mathrm{H}$, Goldstein I. Future targets for female sexual dysfunction. j Sex Med 2016;13:1147-1165.

7. Salonia A, Munarriz RM, Naspro R, Nappi RE, Briganti A, Chionna R, et al. Women's sexual dysfunction: a pathophysiological review. BJU Int. 2004;93:1156-64.

8. Blümel JE, Binfa LE, Cataldo $P A$, et al. Índice de función sexual femenina: un test para evaluar la sexualidad de la mujer. Rev Chil Osbstet Ginecol 2004;69(2):118-125.

9. Mishra VV, Nanda S, Vyas B, Aggarwal R, Choudhary S, Saini SR. Prevalence of female sexual dysfunction among Indian fertile females. J Midlife Health 2016;7:154-8.

10. Stephenson KR, Toorabally N, Lyons L. Further validation of the Female Sexual Function Index: specificity and associations with clinical interview data. J Sex Marital Ther 2016;42:448- 461.

11. Lou WJ, Chen B, Zhu L, Han SM, Xu T, Lang JH, Zhang L. Prevalence and factors associated with female sexual dysfunction in Beijing, China. Chin Med J 2017;130:1389-94.

12. McCool M, Zuelke A, Theurich M. Prevalence of female sexual dysfunction among premonopausal women: a systematic reviwe and meta-analysis of observational studies. Sex Med Rev 2016;4(3):197-212.

13. Rabiepoor S, Khalkhali HR, Sadeghi E. What kind of sexual dysfunction is most common among overweight and obese women in reproductive age? Int J Impot Res 2017;29(2):61-64. 
14. Worsley R, Bell R, Gatoulla p, et al. Pevalence and predictors of low sexual desire, sexually related personal distress, and hypoactive sexual desire dysfunction in a community-based sample of midlife women. J Sex Med 2017;14:675-686.

15. Latif E, Diamond M. Arriving at the diagnosis of female sexual dysfunction. Fertil Steril 2013;100:898-904.
16. Gamal El Atrash, Mohamed H, Hassan A. The assessment of sexual dysfunction in Egyptian women with lower urinary tract symptoms. Arab J Urol 2014;12(3):234-8.

17. Shifren JL, Monz BU, Russo PA, et al. Sexual problems and distress in United States women: prevalence and correlates. Obstet Gynecol 2008;112:970-978.

18. Oksuz E, Malhan S. Prevalence and risk factors for female sexual dysfunction in Turkish women. J Urol. 2006;175:654-58.

\section{AVISO IMPORTANTE}

La Revista Mexicana de Urología se convierte en una publicación solo digital, con todas las ventajas que los medios y dispositivos electrónicos ofrecen. Usted podrá revisar la información mediante el sitio web (www.revistamexicanadeurologia.org.mx) o descargando la app para Android o iPhone.

Para consultar el texto completo de los artículos deberá registrarse por una sola vez con su correo electrónico, crear una contraseña, indicar su nombre, apellidos y especialidad.

Esta información es indispensable para saber qué consulta y cuáles son sus intereses, y poder en el futuro inmediato satisfacer sus necesidades de información. 